\title{
Feasibility of breast conservation surgery in locally advanced breast cancer downstaged by neoadjuvant chemotherapy: A study in mastectomy specimens using simulation lumpectomy
}

\author{
Viswambharan Jaiganesh K, Kadambari D, lyengar Krishnan R, Srinivasan K \\ Departments of Surgery and Pathology, Jawaharlal Institute of Postgraduate Medical Education and \\ Research, Pondicherry, India
}

Correspondence to: Viswambharan Jaiganesh K, E-mail: jaiganeshkv@yahoo.com

\section{Abstract}

BACKGROUND: The response of locally advanced breast cancer (LABC) to neoadjuvant chemotherapy (NACT) offers these patients previously treated by mastectomy, the chance for breast conservation. AIM: This study aims to assess the feasibility of lumpectomy in patients with LABC treated by NACT, with residual tumor $\leq 5 \mathrm{~cm}$. SETTINGS, DESIGN: Single group prospective study from August 2001 to June 2003 in a teaching hospital. MATERIALS AND METHODS: Thirty patients with LABC whose tumors reduced with NACT to $\leq 5 \mathrm{~cm}$ were included. Simulation lumpectomy was performed on the mastectomy specimens to achieve 1 to $2 \mathrm{~cm}$ clearance from tumor and hence margin negativity. Multiple sections of the inked margin were studied. STATISTICAL ANALYSIS: Margin positivity was correlated with patient factors. Chi square test and Fisher's exact test used as appropriate. $P$ value $\leq 0.05$ was considered significant. RESULTS AND CONCLUSIONS: After three cycles of NACT, 4 patients (13\%) had complete clinical response including 2 with complete pathological response. Twenty-two (73\%) showed partial response and 4 , no response. Fourteen out of thirty $(47 \%)$ had tumor involvement of margins. Tumors with post-chemotherapy size $>4 \mathrm{~cm}$ were margin positive in 10/13 (77\%). Tumors with post-chemotherapy size $>3 \mathrm{~cm}$ were margin positive in $13 / 24$ (54\%). Tumors with post-chemotherapy size $\leq 3 \mathrm{~cm}$ were margin negative in $5 / 6$ (83\%). Pre-chemotherapy tumor size and post-chemotherapy tumor size were significantly associated with margin positivity $(P=0.003)$. Tumors in the subareolar location had significantly higher incidence of residual tumor in the nipple areola complex. $(P=0.04)$. Margin positivity of lumpectomy on downstaged tumors can be reduced by removing the nipple areola complex in subareolar tumors and by limiting breast conservation to tumors with post-chemotherapy size $\leq 3 \mathrm{~cm}$.

Key Words: Breast conservation surgery, locally advanced breast cancer, neoadjuvant chemotherapy, downstaging, lumpectomy

\section{Introduction}

The standard treatment of locally advanced breast cancer (LABC) is neoadjuvant chemotherapy (NACT). It has a high rate of success, causing complete clinical response in some patients and reducing the size of the tumor in many patients..$^{[1]}$

Patients with LABC previously treated by total mastectomy with level II axillary clearance could now 
be offered the chance for breast conservation surgery. ${ }^{[1]}$

It is known that the risk of recurrence is related to margin status and within the limits of esthetics, there is consensus that wider margins are always better if achievable. ${ }^{[2]}$

This study is planned with the purpose of demonstrating the feasibility of breast conservation surgery (lumpectomy) by achieving negative surgical margins in simulation lumpectomies performed on the mastectomy specimens of patients with LABC treated by NACT and mastectomy.

\section{Materials and Methods}

This single group prospective study, conducted between August 2001 and June 2003 included patients of LABC with tumor size greater than five $\mathrm{cm}$ and whose treatment plan was neoadjuvant chemotherapy followed by total mastectomy.

Patients unfit for chemotherapy, those requiring preoperative radiation, patients with skin ulceration, those with previous incisional biopsy of the primary tumor and male patients were excluded.

The workup included fine needle aspiration cytology from the primary tumor and palpable axillary lymph nodes, complete blood count with peripheral smear, routine biochemistry and liver function tests, chest Xray, ultrasound of abdomen and electrocardiogram.

For clinical staging, size of the primary tumor was measured over the two longest perpendicular diameters and the product calculated. The number and groups of palpable axillary nodes were noted.

Informed consent was obtained, explaining the nature of disease, treatment options, advantages and side-effects of chemotherapy and need for surgery after chemotherapy. All patients received three cycles of Adriamycin-based chemotherapy intravenously at three weekly intervals (Cyclophosphamide $500 \mathrm{mg} / \mathrm{m}^{2}$, Adriamycin $50 \mathrm{mg} / \mathrm{m}^{2}, 5$-FU $500 \mathrm{mg} / \mathrm{m}^{2}$, Vincristine $1.2 \mathrm{mg} / \mathrm{m}^{2}$; CAFV on Day 1 and $5-\mathrm{FU}$ on Day 8 ). Patients were assessed for side-effects and response of the primary tumor and axilla before every cycle. All postmenopausal patients received Tamoxifen $10 \mathrm{mg}$ twice daily.

Patients were reassessed 21 days after the completion of the third cycle by clinical examination, and ultrasound in patients with complete clinical response. Those whose residual tumor was $>5 \mathrm{~cm}$ were excluded. Those with residual tumor $\leq 5 \mathrm{~cm}$ in longest diameter underwent total mastectomy with level II axillary clearance.

A simulation lumpectomy was performed on the mastectomy specimens with a margin of $1-2 \mathrm{~cm}$ around the palpable tumor. The lumpectomy specimen obtained was marked for orientation of the pathologist, coated with India ink for staining of margins and immersed in formalin for fixation.

Samples were taken from the superior, lateral, inferior and medial margins of the transversely cut central section and from the residual central tumor. The superior and inferior hemispheres were now sectioned in the sagittal plane and margins closest to the tumor were studied. Samples of all quadrants of the breast, any suspicious areas on gross examination and the nipple areola complex were obtained to exclude multicentricity. Examination of axillary fat pad and nodes was done.

Histopathological examination was done for margin involvement by the tumor and described as positive or negative based on the presence or absence of invasive or intraductal carcinoma at the surface of the margin. Samples from the quadrants were studied for any residual invasive carcinoma or insitu carcinoma changes. The nipple areola complex was studied for tumor infiltration. Axillary lymph nodes were examined for metastasis.

Margin positivity was correlated with factors such as age ( $<50$ years), menopausal status, site of tumor, prechemotherapy tumor size, clinical response, and postchemotherapy tumor size.

Statistical Analysis-Chi square test, Fisher's exact test and student's $t$ test were used as appropriate. A $P$ value of less than or equal to 0.05 was considered statistically significant. The Statistical Package for Social Sciences (SPSS, Chicago, Illinois, USA) software version 10.0 was utilized for data analysis.

The study protocol was approved by the hospital's ethics committee.

\section{Results}

Forty patients were initially included in the study. Only 36 completed three cycles of chemotherapy. Of these 6 patients showed no response, their tumors did not reduce to a size $\leq 5 \mathrm{~cm}$ and they were not considered for the rest of the study. 
The patients' age ranged from 30-72 years (mean $=48$ years) and all were multiparous. Thirteen patients $(43 \%)$ were premenopausal and 17 patients $(57 \%)$ were postmenopausal. Tumors were right-sided in 16 and left-sided in 14. Upper outer quadrant tumors predominated, 18/30. Five patients had central quadrant tumors. Sixty per cent patients presented within 4 months of onset of symptoms (lump in breast). No tumor was fixed to the pectoralis major or the chest wall. There was no detectable distant metastasis. Twenty-one out of thirty $(70 \%)$ had T3 tumors whereas $9 / 30$ had T4 tumors. Axillary nodal status was N1 in 28 patients $(93 \%)$ and $\mathrm{N} 2$ in 2 patients $(7 \%)$. Ttwenty-one patients had Stage IIIa disease and 9 had Stage IIIb disease. The size of the primary tumor ranged from $5-9 \mathrm{~cm}($ mean $=7.1 \mathrm{~cm})$. Axillary nodes ranging from 1-3 in number were palpable in all. All patients tolerated chemotherapy without major complications, alopecia and vomiting being the commonest side-effects.

Of 30 patients 4 (13\%) had complete clinical response, $22(73 \%)$ had partial response and 4 (13\%) no response. Complete pathological response was seen in $2 /$ 4 of the patients with complete clinical response.

There was a significant association between prechemotherapy tumor size and histopathological margin status $(P=0.003)$ (Table 1$)$ and a significant difference in mean tumor size in patients with positive histopathological margin $(7.5 \pm 0.76 \mathrm{~cm})$ and negative histopathological margin $(6.63 \pm 1.02 \mathrm{~cm})$ by students ' $\mathrm{t}$ ' test $(P=0.01)$. The size of the tumor decreased in response to chemotherapy in all the patients. Mean post-chemotherapy tumor size was $3.8 \mathrm{~cm}$.

There was a significant association between postchemotherapy tumor size and histopathological margin status $(P=0.003)$ (Table 1$)$.

In tumors with post-chemotherapy tumor size $>4 \mathrm{~cm}$, $10 / 13(77 \%)$ were margin positive and $3 / 13(23 \%)$ were margin negative whereas in tumors with postchemotherapy size $>3 \mathrm{~cm}, 13 / 24(54 \%)$ were margin positive and $11 / 24(46 \%)$ were margin negative. In tumors with post-chemotherapy size $<3 \mathrm{~cm}, 1 / 6(17 \%)$ were margin positive and $5 / 6(83 \%)$ were margin negative.

Clinical examination of the axilla revealed a complete response in 10/30 (33\%) and an incomplete response in 20/30 (67\%). Pathological examination showed metastasis in $20 / 29(69 \%)$ and no metastasis in $9 / 29(31 \%)$.

All patients underwent total mastectomy and 29

\section{Table 1: Analysis of patient and tumor characteristics associated with histopathological margin status}

\begin{tabular}{|c|c|c|c|c|}
\hline \multirow[b]{2}{*}{ Characteristics } & \multicolumn{4}{|c|}{ Margin status } \\
\hline & & Positive & Negative & $P$ value \\
\hline \multirow[t]{2}{*}{ Age (Years) } & $<50$ & 8 & 9 & \\
\hline & $\geq 50$ & 6 & 7 & 0.961 \\
\hline \multirow[t]{2}{*}{ Menopausal status } & Premenopausal & 6 & 7 & \\
\hline & Postmenopausal & 8 & 9 & 0.961 \\
\hline \multirow[t]{2}{*}{ Tumor location } & Subareolar & 4 & 1 & \\
\hline & Other quadrants & 10 & 15 & 0.419 \\
\hline \multirow[t]{2}{*}{ T stage } & T3 & 10 & 11 & \\
\hline & $\mathrm{T} 4$ & 4 & 5 & 0.873 \\
\hline \multirow[t]{2}{*}{ AJCC stage } & IIla & 10 & 11 & \\
\hline & IIlb & 4 & 5 & 0.873 \\
\hline \multirow[t]{2}{*}{ Clinical response } & Complete & 1 & 3 & \\
\hline & Incomplete & 13 & 13 & 0.35 \\
\hline Pre-chemotherapy & $5.1-7.0$ & 5 & 14 & \\
\hline Tumor size $(\mathrm{cm})$ & 7.1-9.0 & 9 & 2 & 0.003 \\
\hline Post-chemotherapy & $>4$ & 10 & 3 & \\
\hline Tumor size $(\mathrm{cm})$ & $\leq 4$ & 4 & 13 & 0.0036 \\
\hline Post-chemotherapy & $>3$ & 13 & 11 & \\
\hline Tumor size $(\mathrm{cm})$ & $\leq 3$ & 1 & 5 & 0.17 \\
\hline Axillary lymph node & Positive & 11 & 9 & \\
\hline metastasis & Negative & 3 & 6 & 0.28 \\
\hline
\end{tabular}

underwent level II axillary clearance. One patient did not undergo axillary clearance due to fibrosis of axilla. Simulation lumpectomy margins were free from tumor on gross examination. In $4 / 30$ cases the skin overlying the tumor showing fixity had to be removed with $1 \mathrm{~cm}$ margin. Skin margins were not involved on histopathological examination. The nipple areola complex was sacrificed in one case since the areolar skin appeared to be involved by the tumor. There was no visible or palpable residual tumor in any of the mastectomy specimens.

Histopathological examination of the margins showed positive margins in $14 / 30(47 \%)$ and negative margins in $16 / 30(53 \%)$. Of the 14 patients who had peripheral margin involvement, 10 had single peripheral margin involved. Seven were partial responders, 2 were in the no response category and one was in the complete response category. A clearance of 1 to $2 \mathrm{~cm}$ was achieved in 7/10 of these cases. Four patients had two peripheral margins involved and all were in the partial response category. A clearance margin of 1 to $2 \mathrm{~cm}$ was achieved in three of these cases.

Tumor histology was infiltrating ductal carcinoma in $29 / 30$ and infiltrating lobular carcinoma in one. In 2 
patients with complete pathological response, the lumpectomy specimen did not reveal any tumor. The remaining breast tissue in the mastectomy specimen did not show any invasive carcinoma or any ductal carcinoma in situ (DCIS).

The nipple areola was positive in 6/30 (20\%). Of these 3 were subareolar tumors, $3 / 6(50 \%)$. There was a significant association between tumor location and histopathological status of the nipple areola complex (Table 2) $(P=0.04$ by Fischer's exact test $)$.

\section{Discussion}

The overall objective response of the primary tumor was $87 \%$ with a complete clinical response of $13 \%$. Machiavelli et $\mathrm{al}^{[3]}$ in their study of 148 patients with LABC recorded objective response in $71 \%$. Primary tumor response was assessed by clinical examination. Herrada et $\mathrm{a}^{[4]}$ demonstrated that physical examination correlated best with pathological findings in the measurement of primary tumor.

There was a significant association between prechemotherapy tumor size, post-chemotherapy tumor size and histopathological margin status. Tumor size was found to be associated with positive surgical margins by Peterson et $\mathrm{al}^{\left[{ }^{[5]}\right.}$ Park et $\mathrm{al}^{\left[{ }^{[6]}\right.}$ and Tartter et $\mathrm{al}^{[7]}$ by univariate analysis. Tartter et $\mathrm{al}^{[7]}$ and Luu et al ${ }^{[8]}$ demonstrated by multivariate analysis also, that tumor size showed a significant correlation with surgical margins.

In tumors with post-chemotherapy size $>4 \mathrm{~cm}, 10 / 13$ (77\%) were margin positive, and $13 / 24$ (54\%) were margin positive in tumors with post-chemotherapy size $>3 \mathrm{~cm}$. In tumors with post-chemotherapy size $<3 \mathrm{~cm}$ $1 / 6(17 \%)$ were margin positive and $5 / 6(83 \%)$ were margin negative.

Therefore a smaller post-chemotherapy size is found to give lesser margin positivity. The previous recommendation for breast conservation surgery was for tumors up to $4 \mathrm{~cm}$ or those with residual tumor size $<$ $5 \mathrm{~cm} .{ }^{[9]}$ Cosmetic results are more acceptable with

Table 2: Correlation of histopathological status of the nipple areola complex with tumor location

\begin{tabular}{lccc} 
& \multicolumn{3}{c}{ Nipple areola } \\
\cline { 2 - 4 } Tumor location & Positive & Negative & Total \\
\hline Subareolar & 3 & 2 & 5 \\
\hline Other quadrants & 3 & 22 & 25 \\
\hline Total & 6 & 24 & 30 \\
\hline
\end{tabular}

smaller tumors. ${ }^{[10-12]}$ Cosmetic outcome could not be evaluated in our study since surgery was done on specimens.

Various authors have studied patient and tumor characteristics associated with positive surgical margins in patients with invasive ductal carcinoma treated with primary breast conservation therapy. ${ }^{[5-8,13-15]}$ Those which were significant included younger age, DCIS, extensive intraductal component, positive family history, lymphovascular invasion, axillary node positivity status and large tumor size.

In this study age, menopausal status, quadrant location, T stage, AJCC stage, clinical response to NACT and axillary lymph node status were not found to be significantly associated with histopathological margin positivity.

Margin positivity may be either due to technical error in dissection or due to the response of the tumor to chemotherapy, leaving behind islands of tumor cells while it shrinks. This phenomenon was explained by Hunt et $\mathrm{al}^{[11]}$ and Moneer et $\mathrm{a}^{[16]}$ as due to irregular tumor angiogenesis or tumor polyclonality, resulting in cell populations with varying exposure and susceptibility to the cytotoxic agents.

Holland et $\mathrm{al}^{[17]}$ in their classical pathologic study estimated that if conservative surgery was done with a $2-\mathrm{cm}$ margin, infiltrating disease would be left behind in $12 \%$ and $\mathrm{ca}$ in situ in $25 \%$. With a $3-\mathrm{cm}$ margin these values were $8 \%$ and $9 \%$ and with $4 \mathrm{~cm}$ the values were $5 \%$ and $5 \%$. Since a $1-2 \mathrm{~cm}$ margin was given in our study, the almost $50 \%$ positivity of the margins has been contributed to, at least in part, by the process of tumor segmentation.

In our study, the rest of the breast tissue of the mastectomy specimen did not show any evidence of invasive carcinoma or ductal carcinoma in situ in spite of sampling from all quadrants, any suspicious areas and the nipple areola complex. Moneer et $\mathrm{al}^{[16]}$ had 9 no response cases and tumor multifocality was observed in only 1 case $(11 \%)$ whereas $20 / 29(70 \%)$ of the partial response cases had tumor foci in the vicinity of the main lesion. This was statistically highly significant.

Veronesi et $\mathrm{al}^{[18]}$ in their study of 226 patients found histological multifocality in the primary in 37 patients (16\%). In $22(60 \%)$ of these cases, pre-chemotherapy mammography showed a carcinoma associated with microcalcifications. Multifocality was observed more frequently in larger tumors, probably because the 
tumors had not been uniformly destroyed by chemotherapy.

The nipple areola complex was positive for tumor in 6/ $30(20 \%)$. Of these 3 were central quadrant tumors. There was a significant association between tumor location and the histopathological status of the nipple and areola. Gajdos et al ${ }^{[19]}$ in their study of 95 patients with subareolar cancer showed that subareolar cancers can be successfully treated with breast conservation surgery provided adjuvant radiotherapy is always given, and that clinical involvement of the nipple areola complex is associated with high risk of local failure when treated with breast conservation without radiation therapy.

Since $3 / 5$ of the central quadrant tumors were showing nipple areola complex involvement, it might be recommended that the nipple and areola be removed in cases of breast conservation in subareolar tumors.

In conclusion, the following methods may be adopted to reduce margin positivity in breast conservation surgery performed on locally advanced breast cancer downstaged with neoadjuvant chemotherapy.

1. Removal of the nipple areola complex in subareolar tumors.

2. Limiting breast conservation surgery to tumors with a post-chemotherapy size less than $3 \mathrm{~cm}$.

In patients showing partial response and/or those with a larger tumor size at presentation, wider margins may be useful during lumpectomy for removing tumor foci left behind due to tumor segmentation.

The question also arises whether additional cycles of NACT and/or use of taxanes will improve the response and thereby lessen the margin positivity.

Further studies with larger numbers, incorporating use of radiological methods for tumor measurement and marking and cytological methods for intraoperative margin assessment will be required to make recommendations for breast conservation surgery in locally advanced breast cancer downstaged with neoadjuvant chemotherapy.

\section{References}

1. Gianni L, Valagussa P, Zambetti M, Moliterni A, Capri G, Bonadonna G, et al. Adjuvant and neoadjuvant treatment of breast cancer. Semin Oncol 2001;28:13-29.

2. Schwartz GF, Hortobagyi GN . Proceedings of the Consensus conference on neoadjuvant chemotherapy in Carcinoma of the Breast, April 26-28, 2003, Philadelphia, Pennsylvania. Cancer 2004; 100:2512-32.

3. Machiavelli MR, Romero AO, Perez JE, Lacava JA, Dominguez ME, Rodriguez R, et al. Prognostic significance of pathological response of primary tumor and metastatic axillary lymph nodes after neoadjuvant chemotherapy for locally advanced breast carcinoma. Cancer J Sci Am 1998;4:125-31.

4. Herrada J, lyer RB, Atkinson EN, Sneige N, Buzdar AU, Hortobagyi $\mathrm{GN}$. Relative value of physical examination, mammography and breast sonography in evaluating the size of the primary tumor and regional lymph node metastases in women receiving neoadjuvant chemotherapy for locally advanced breast carcinoma. Clin Cancer Res 1997;3:1565-9.

5. Peterson ME, Schultz DJ, Reynolds C, Solin LJ, et al. Outcomes in breast cancer patients relative to margin assessment after treatment with breast conserving surgery and radiation therapy: The University of Pennysylvania experience. Int J Radiat Oncol Biol Phys 1999;43:1029-35.

6. Park CC, Mitsumori M, Nixon A, Recht A, Connolly J, Gelman R, et al. Outcome at 8 years after breast-conserving surgery and radiation therapy for invasive breast cancer: Influence of margin status and systemic therapy on local recurrence. J Clin Oncol 2000; 18: 1668-75.

7. Tartter PI, Kaplan J, Bleiweiss I, Gajdos C, Kong A, Ahmed S, et al. Lumpectomy margins, re-excision and local recurrence of breast cancer. Am J Surg 2000;179:81-5.

8. Luu HH, Otis CN, Reed WP Jr, Garb JL, Frank JL. The unsatisfactory margin in breast cancer surgery. Am J Surg 1999; 178:362-6.

9. Kuerer HM, Hunt KK, Newman LA, Ross MI, Ames FC, Singletary SE. Neoadjuvant chemotherapy in women with invasive breast carcinoma:conceptual basis and fundamental surgical issues. J Am Coll Surg 2000; 190:350-63.

10. Singletary SE. Neoadjuvant chemotherapy in the treatment of stage II and stage III breast cancer. Am J Surg 2001;182:341-6.

11. Hunt KK, Buzdar AU. Breast conservation after tumor downstaging with induction chemotherapy. In: Singletary SE, editor. Breast Cancer, $1^{\text {st }}$ Ed. Springer Verlag Inc. 1999.

12. Rouzier R, Mathieu M, Sideris L, Youmsi E, Rajan R, Garbay J, et al. Breast conserving surgery after neoadjuvant anthracycline based chemotherapy for large breast tumors. Cancer 2004; 101:918-25.

13. Obedian E, Haffty BG. Negative margin status improves local control in conservatively managed breast cancer patients. Cancer J Sci Am 2000;6:28-33.

14. Horiguchi J, linoY, Takei H, Maemura M, Yokoe T. Niibe H, et al. Surgical margin and breast recurrence after breast- conserving therapy. Oncol Rep 1999;6:135-8.

15. Borger J, Kemperman H, Hart A, Peterse H, van Dongen J, Bartelink $\mathrm{H}$. Risk factors in breast- conservation therapy. J Clin Oncol 1994; 12:653-60.

16. Moneer M, El-Didi M, Khaled H. Breast conservative surgery: Is it appropriate for locally advanced breast cancer following downstaging by neoadjuvant chemotherapy? A Pathological assessment. Breast 1999;8:315-9.

17. Holland R, Veling SH, Mravunac M, Hendriks JH, et al. Histologic multifocality of Tis, T1-2 breast carcinomas. Implications for clinical trials of breast- conserving surgery. Cancer 1985;56:979-90.

18. Veronesi U, Bonadonna G, Zurrida S, Galimberti V, Greco M, Brambilla $\mathrm{C}$, et al. Conservation surgery after primary chemotherapy in large carcinomas of the breast. Ann Surg 1995;222:612-8.

19. Gajdos C, Tartter PI, Bleiweiss IJ. Subareolar breast cancers. Am J Surg 2000; 180: 167-70. 